\title{
Interaction Techniques for Unreachable Objects on the Touchscreen
}

\author{
Sunjun Kim HCI \\ Lab, KAIST Daejeon, \\ South Korea \\ kuaa.net@gmail.com
}

\author{
Jihyun Yu NC Lab, \\ KAIST Daejeon, \\ South Korea \\ yjh0502@gmail.com
}

\author{
Geehyuk Lee HCI \\ Lab, KAIST Daejeon, \\ South Korea \\ geehyuk@gmail.com
}

\begin{abstract}
Large-screen mobile devices have recently been introduced. While they can display more information on the screen, they have raised the issue of thumb reachability during one-handed use. To solve this problem, we designed four factorial combinations of two triggering techniques (Edge and Large touch) and two selection techniques (Sliding screen and Extendible cursor). A prototype realizing the four interaction techniques was implemented, and a user study was conducted to examine the benefits and problems faced while using these techniques in both portrait and landscape orientations. User study exhibited a significant advantage of Edge triggering with Extendible cursor technique. Also, we collected meaningful comments from the user interview.
\end{abstract}

\section{Author Keywords}

Short-thumb problem, one-handed interaction, largescreen mobile devices, interaction techniques

\section{ACM Classification Keywords}

H.5.2. User Interfaces: Input Devices and Strategies, Interaction Styles, Screen Design; D.2.2 User Interface

\section{INTRODUCTION}

Large touchscreen devices have recently been introduced. They can display more information owing to their large screens, but they are difficult to operate with one hand. During one-handed usage, users need to employ their thumb to manipulate objects on the screen. However, it is difficult for the thumb to cover the complete screen area, as shown in Figure 1.

Users may employ both hands to manipulate such a largescreen device; however, they are often forced to use the device with one hand, for example, when the user is in motion and one hand is occupied by a bag. In one-handed interaction, the thumb is the only manipulating pointer, and therefore, the reachable area of the screen is restricted by thumb length. According to the study by Katre (Katre et al., 2008), the average thumb length of users in India was $6.9 \mathrm{~cm}$ (approx. 2.7"). Although this number may vary by race, it seems to be generally true that it is hard to operate a large touchscreen device with only one hand. In order to reach an object at the farther side of the screen,

Permission to make digital or hard copies of all or part of this work for personal or classroom use is granted without fee provided that copies are not made or distributed for profit or commercial advantage and that copies bear this notice and the full citation on the first page. To copy otherwise, or republish, to post on servers or to redistribute to lists, requires prior specific permission and/or a fee.

OZCHI'12, November 26-30, 2012, Melbourne, Victoria, Australia Copyright 2012 ACM 978-1-4503-1438-1/12/11 ..\$10.00. users can readjust their grip, but this is often an unsafe action because of the risk of dropping the device. Alternatively, they may switch to a two-handed mode, but this may not always be possible or may be time consuming. In this paper, we propose four factorial combinations of two triggering techniques (Edge and Large touch) and two selection techniques (Sliding screen and Extendible cursor) to solve this problem.

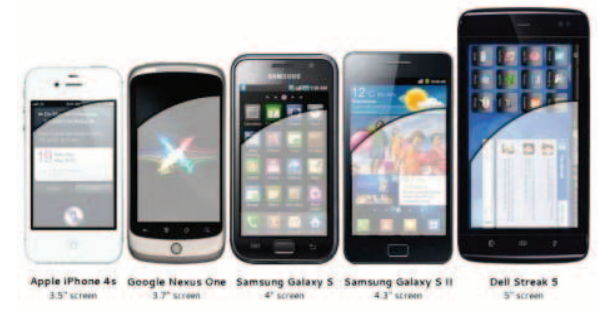

Figure 1. The reach of the thumb is shown with screen sizes varying from 3.5 -inches to 5 -inches.

\section{RELATED WORKS}

ThumbSpace (Karlson et al., 2007) introduced onehanded thumb interaction for small targets that are spread out wide on large screens, which is identical to our problem description. It solved the problem by squeezing the screen into a small proxy screen. However, this inherited and reproduced the fat finger problem associated with small screens. Oh et al. (Oh et al., 2010) condensed icons so that they would fit into the reachable area. They also utilized a cursor to select targets positioned at the farther end of the screen. These two studies successfully solved the short-thumb problem. However, the triggering method-for example, pressing a physical button-was not well integrated with the direct manipulation paradigm of the touchscreen.

Perry et al. (Perry et al., 2008) evaluated one-handed thumb tapping interactions for various target positions, target sizes, and situations. Their result shows that better accuracy is obtained for objects positioned at the edge of the screen. BezelSwipe (Roth et al., 2009) also takes advantage of the device edge, which is easily and accurately accessible. These works inspired us to use the Edge triggering technique in our work. TapTap and MagStick (Roudaut et al., 2008) facilitate one-handed interaction on small touchscreen devices. Although they do not support large-screen devices, the pointing stick approach on MagStick influenced our Extendible Cursor design.

\section{DESIGNING INTERACTION}

Our goal is the design of a new support interface to solve the short-thumb problem. Our approach involves two 
steps: triggering and selection. If a user performs triggering gestures (trigger), the selection interface becomes ready to use. The user can then select a target object (selection).

Several design goals were established: The interface should be orthogonal to the usual touch and multi-touch interactions and gestures. It should also be intuitive and easy to learn and use. Finally, it should have acceptable performance, i.e., it should not be too slow in comparison to regular touch interaction.

Two triggering methods were designed: Edge and Large touch. Edge is a swiping gesture from the edge across the screen, and Large touch is a touch gesture using a wide area of the finger, for example, the pad of the thumb. Two selection methods were subsequently designed: Slidingscreen and Extendible cursor. In Sliding-screen, the user can freely move the entire screen, which allows them to move a target closer to the thumb. In Extendible cursor, a circular cursor is generated and it multiplies thumb movements similar to the manner in which a lever multiplies the effect of an action.

There are two triggering methods (Edge and Large touch) and two selection methods (Sliding Screen and Extendible Cursor). Thus, four combinational configurations were proposed by combining two triggers with two selection methods. Figure 2 illustrates the configurations and their names: $E S, L S, E C$, and $L C$. For all support interfaces, Direct touch method was always provided by default: participants can choose whether to use support interface or not.
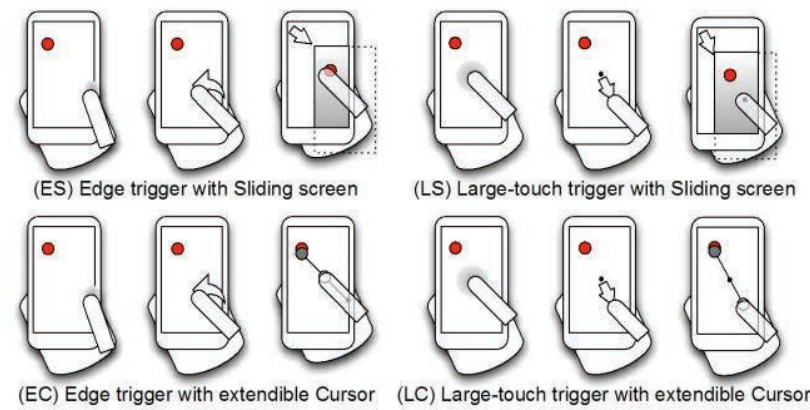

Figure 2. Four design configurations and their behaviors. In extendible cursor, a small black dot is the reference point, and the large grey dot is the circular cursor.

Depending on the triggering method, different behaviors were applied in the selection step. In $L S$ and $E C$, a screen or a cursor moves in the same direction as the finger movement. On the other hand, in $E S$ and $L C$, they move in the opposite direction. In $E S$, the actual triggering and selection are accomplished with a single gesture. Because this gesture starts from the edge of a device, there is no possibility of pulling the screen toward the edge. For this reason, the screen should move in the opposite direction. In $L C$, the situation in which a user failed to reach an object was assumed. In this situation, the thumb is already stretched as far as possible. To reduce the amount of finger movement, the cursor was designed to move in the opposite direction.

\section{PILOT STUDY}

A pilot study was designed and the task completion time, accuracy, and user preference were measured. Our design was implemented on the Samsung I9100 (Galaxy S2) Smartphone with a 4.3 " capacitive multi-touch screen with a $480 \times 800$ screen resolution. Eight participants (7 Male, 1 Female, Ages 18-28) were used. All participants were right-handed and had experience with touchscreen based smartphones. The experiment required forty minutes per participant, and they were paid five dollars upon the completion of the experiment.

\section{Experiment Design}

Each participant was asked to hold the device with the dominant hand and manipulate it only with the thumb. They were asked to perform target selection tasks. The target was a circular icon with a radius of $50 \mathrm{px}(1.09 \mathrm{~cm})$, the usual icon size in the Android OS. The screen was divided into $3 \times 4$ size grids, and a target appeared at the center of a grid in a random order. Targets appeared four times for each grid, whereby each session consisted of 48 target selection tasks. When a participant succeeded in correctly selecting a target, he or she received haptic feedback through a vibration motor and the next target was immediately generated. If a participant failed to select a target, no feedback was provided to ensure that the participant would try again.

Five configurations (Direct, $E S, L S, E C$, and $L C) \times$ two types of orientations (portrait, landscape) were provided, thus $5 \times 2$ within-subject experiment was conducted. The order was randomized. A tutorial session was provided before each session. Participants were given the chance to tune several parameters during the tutorial sessions: Touch area threshold for Large touch, and ControlDisplay gain (CD gain) for the Sliding Screen and Extendible cursor. By tweaking the Touch area threshold, participants could adjust the width of the area they needed to touch to activate Large touch. Likewise, by tweaking $C D$ gain, participants could adjust how fast the screen or the cursor moved. After the tutorial, participants performed 48 target selection tasks. They were asked to perform each task as accurately and quickly as possible.

After finishing all sessions, participants were asked to complete the satisfaction score (a seven-point Likert scale) for all ten conditions. Finally, the participants were interviewed for ten minutes. They were asked for any opinions about our interfaces, such as the reasons for their answers, frustrating experiences, and suggestions for each configuration.

\section{Result}

For each participant, 480 selection logs (48 trials $\times 5$ configurations $\times 2$ orientations) and user satisfaction results were collected. Based on the logs, task completion time, accuracy, and error count were collected for all sessions. Task accuracy was defined as the distance from the center of a target and a selected location. Unfortunately, we could analyze logs from seven participants because the log of P1 was corrupted. 
Task completion time

Task completion time was defined as the elapsed time between a target appearance and a successful target selection. Figure 3 illustrates the completion time. We analyzed results by repeated-measure ANOVA. The configuration did exhibit a significant effect on task completion times $(\mathrm{F}(4,24)=7.415, \mathrm{p}<.001)$. With post hoc pairwise t-test with a bonferroni correction, only $L S$ $(\mathrm{p}<.05)$ was significantly slower than Direct and other pairs resulted in no significance. The orientation also did exhibit a significant effect on task completion times $(\mathrm{F}(1,6)=29.282, \mathrm{p}<0.01)$, with portrait resulting in $1198.91 \mathrm{~ms} \quad(\mathrm{SD}=62.79)$ and landscape resulting in $1532.17 \mathrm{~ms} \quad(\mathrm{SD}=94.88)$. There was no significant configuration*orientation interaction $(\mathrm{F}(4,24)=1.393$, n.s., $\mathrm{p}=.266)$.

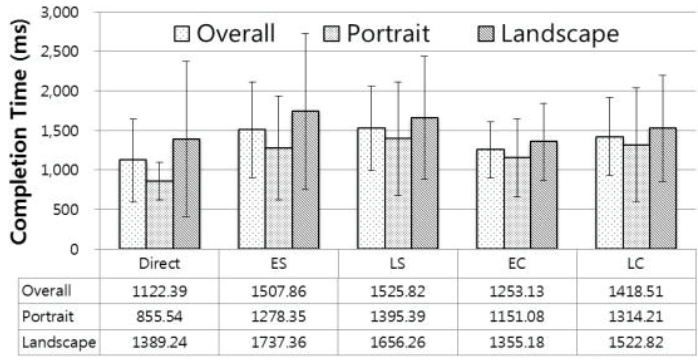

Figure 3. Average completion times (bars: $95 \% \mathrm{CI}$ ).

\section{Accuracy and Error count}

Task accuracy was defined as the distance from the center of a presented target and a selected location. Figure 4 illustrates the accuracy. We analyzed results by repeatedmeasure ANOVA. The configuration did exhibit a significant effect on accuracy $(\mathrm{F}(4,24)=6.997, \mathrm{p}=.001)$. With post hoc pairwise t-test with a bonferroni correction, $E C$ was significantly more accurate than $E S(\mathrm{p}=.003)$ and there was a trend between $E C$ and $L S(\mathrm{p}=.084)$. Other pairs resulted in no significance. The orientation also did exhibit a significant effect on task completion times: Portrait exhibited more accurate result than landscape. $(\mathrm{F}(1,6)=37.174, \mathrm{p}=.001)$. There was a significant configuration*orientation interaction $(\mathrm{F}(4,24)=3.38$, $\mathrm{p}=.025)$. Because we observed the interaction, we also compared configurations within each orientation by pairwise t-test with bonferroni correction. Within portrait orientation, no configuration pairs exhibited any significance. Within landscape orientation, EC was significantly more accurate than $E S$ and $L S$ (both $\mathrm{p}<.05$ ).

Error count was defined as the number of erroneous selections during one session (48 tasks). Figure 5 illustrates the error count. We analyzed results by repeated-measure ANOVA. The configuration did exhibit a significant effect on accuracy $(\mathrm{F}(4,24)=40.60, \mathrm{p}<.001)$. With post hoc pairwise t-test with a bonferroni correction, all Sliding Screen methods (ES and $L S)$ exhibited significantly more errors than Direct and Extendible Cursor methods (EC and $L C, \quad \mathrm{p}<.01)$. No significant differences were observed between Direct and Extendible Cursor methods. The orientation also did exhibit a significant effect on task completion times $(\mathrm{F}(1,6)=48.06$, $\mathrm{p}<.001)$, with portrait resulting in 21.74 errors $(\mathrm{SD}=3.39)$ and landscape resulting in 37.67 errors $(\mathrm{SD}=4.263)$.
Configuration*orientation interaction showed a trend $(\mathrm{F}(4$, $24)=2.529, \mathrm{p}=.067)$. We again compared configurations within each orientation by pairwise t-test with bonferroni correction. Within portrait orientation, $E C$ resulted in significantly low errors than others except Direct (all $\mathrm{p}<.05)$. $L S$ exhibited marginal results with Direct $(\mathrm{p}=.092)$ and $L C(\mathrm{p}=.088) . E S$ exhibited significant higher errors than Direct and EC $(\mathrm{p}<.05)$. Within landscape orientation, Sliding Screen methods exhibited significantly more errors than Direct and Extendible Cursor methods (all $\mathrm{p}<.05$ ).

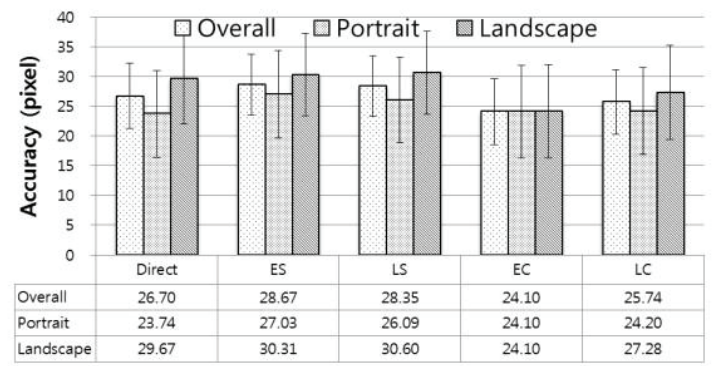

Figure 4. Average accuracy (error bars: $95 \% \mathrm{CI}$ ).

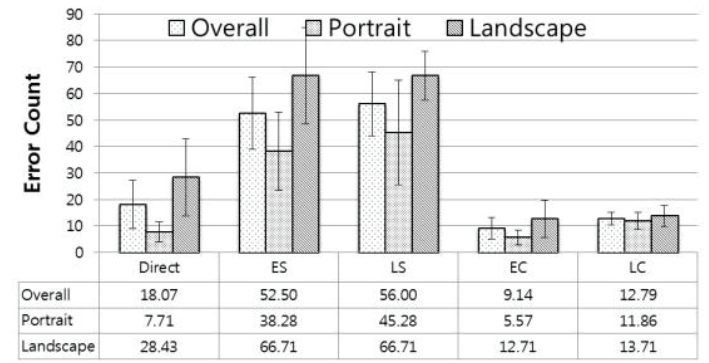

Figure 5. Average error count (error bars: 95\% CI).

Method preference

Figure 6 shows the method preference. As we mentioned in design section, participants could choose between the support interface and direct touch. Therefore, by observing them, it could be verified that our support interfaces are preferred for hard-to-reach targets. The value in the figure represents the portion of the support interface selected. For example, 0 (black) means all targets are selected by a direct touch, while 1 (white) means all targets are selected by a support interface. Our proposed methods were preferred in the upper left sections of the screens, which are harder to tap with one hand.

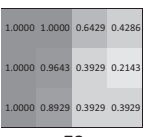

ES

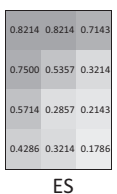

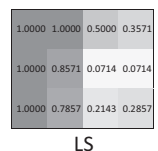

LS

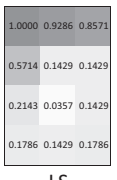

Figure 6. Method preference.

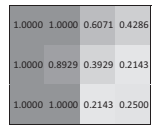

EC
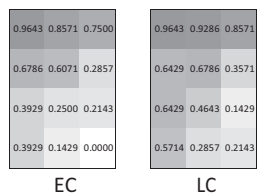

User satisfaction

Finally, the user satisfaction score was examined (Figure 7). We compared results using Wilcoxon signed-rank test. We first compared orientations. Portrait was significantly preferred. $(Z=2.25, p<.05)$ Next, we compared configuration pairs. EC was significantly 
preferred over Direct $(\mathrm{Z}=2.97, \mathrm{p}<.01), L S \quad(\mathrm{Z}=2.00$, $\mathrm{p}<.05)$, and $L C(\mathrm{Z}=3.17, \mathrm{p}<.01)$. Within landscape orientation, all configurations were significantly preferred over Direct (EC-Direct, $\mathrm{Z}=3.35, \mathrm{p}<.001 ;$ ES-Direct, $\mathrm{Z}=2.41, \mathrm{p}<.05$, LS-Direct, $\mathrm{Z}=2.32, \mathrm{p}<.05, L C$-Direct, $\mathrm{Z}=1.84$, marginal, $\mathrm{p}=.065$ ). Within portrait orientation, no pair showed any significance.

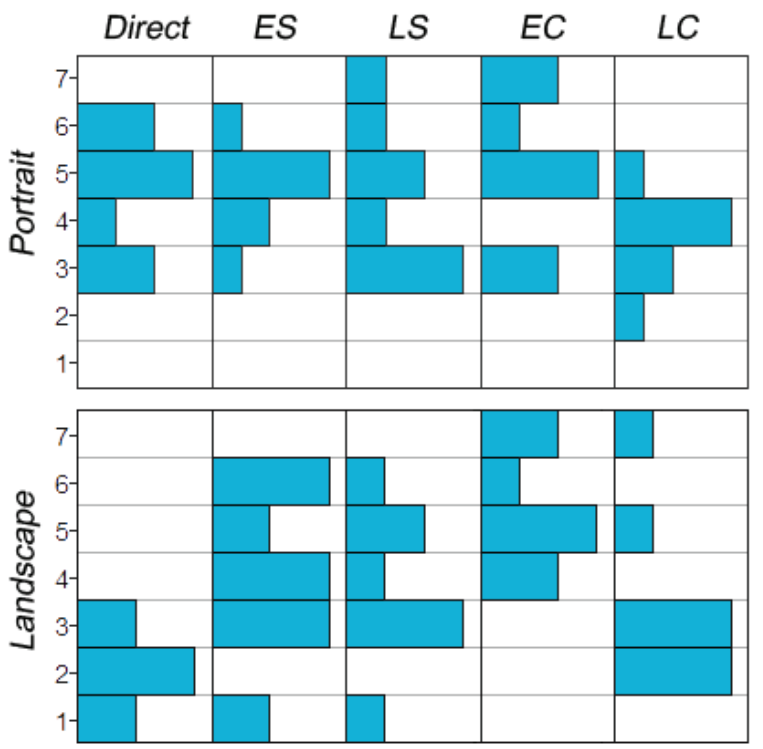

Figure 7. User satisfaction score histograms

\section{Interview comments}

All participants reported that the Direct method with the landscape orientation was extremely uncomfortable. They reported that it was impossible to select a target without changing the grip. They could feel more stability with the landscape orientation while using the proposed techniques. For portrait orientation, P1 through P6 reported that they did not have any problem using the Direct approach. They felt that the entire screen was manageable. Exceptionally, P7 was a woman and P8 was a high-school student, and they felt some difficulties because they had small hands relative to other participants. If the experiment were to be conducted using a larger device, such as the Samsung Galaxy Note or Dell Shriek, the problem would be more noticeable even for portrait orientation.

Edge was generally more preferred as a trigger. Most users reported that they could explicitly observe the activation by the trigger. Comparatively, most participants reported that Large touch was unreliable. This is because the size of the touch area varies with the screen position, given that the touch area increases when the thumb moves further from its usual position. Accordingly, P2, P4, P5, and $\mathrm{P} 7$ reported that the EC condition was most comfortable. In particular, P5 and P7 gave the maximum scores in the satisfaction survey for both the portrait and landscape groups. In contrast, the $L C$ condition showed relatively lower scores. Participants said that the $L C$ configuration was not easy to adapt to reverse movement of cursor. Similarly, P3 was confused with $E S$ condition because it moved in reverse. In contrast, P7 felt that the reverse movement was more preferable. Other users had no comments about the direction of movement. No problems were expected, considering that users would have sufficiently adapted to the interface. The $L S$ condition was generally acceptable; however, most complaints were raised due to the unreliability of Large touch.

Some comments were gathered about the implementation details. A sliding screen should prevent the screen from disappearing entirely. A cursor is risky, because the retry cost is higher. A variable CD gain was proposed instead of a fixed one. It would be beneficial to show the lever along with a cursor. It sometimes requires time to decide whether to use regular direct touch or the support interface.

\section{CONCLUSION}

Four one-handed interaction techniques were designed and evaluated to solve the short-thumb problem. All new methods were preferred, especially with Landscape orientation. Notwithstanding the additional burden of support interfaces, most of them showed similar task completion time than the Direct method. In terms of performance, Extendible Cursor methods showed the same or better performance than the Direct method in terms of the accuracy and error count. Sliding screen methods were more erroneous. Reverse movements such as $L C$ and $E S$ are not very favored. All proposed methods were shown to have a significant advantage for far targets and to be especially helpful in landscape orientations. Most participants agreed that grips were more stable with the new methods. Overall, Edge triggering with Extendible cursor $(E C)$ is proven to the best combination. This work is a formative phase with a focus on identifying problems. We have found valuable clues for designing a new interface. Based on these results, the current prototype is being refined for a more conclusive evaluation of the proposed ideas.

\section{ACKNOWLEDGEMENT}

This work was supported by the IT R\&D program of MKE/KEIT. [10039161, Development of core technologies for high-physicality control interfaces and personalized intelligent user interfaces based on Smart TV user experience]

\section{REFERENCES}

Karlson, A. and Bederson, B. ., ThumbSpace: generalized one-handed input for touchscreen-based mobile devices. In Proc. INTERACT 2007, Springer-Verlag (2007), 324-338.

Katre D. S., One-Handed Thumb Use on Smart Phones by Semi-literate and Illiterate Users in India: A Usability Report with Design Improvements for Precision and Ease. In Proc. Workshop on Cultural Usability and Human Work Interaction Design. Design for All Institute of India 3 (2008), 72-85.

Oh. S., Design and evaluation of methods for aiding one-hand input in mobile touch screen device, In Proc. The fall workshop of Ergonomics society of Korea, (2010), CD-ROM

Perry, K. B. and Hourcade, J. P., Evaluating One Handed Thumb tapping on mobile touchscreen devices. In Proc. GI 2008, Canadian Information Processing Society (2008), 57-64

Roth, V. and Turner, T., Bezel swipe: conflict-free scrolling and multiple selection on mobile touch screen devices. In Proc. CHI 2009. ACM Press (2009), 1523-1526.

Roudaut, A. and Huot, S. and Lecolinet, E., TapTap and MagStick: improving one-handed target acquisition on small touch-screens. In Proc. AVI 2009, ACM Press (2008), 146-153 